\title{
Snap Forecast for Net Image Reranking using Multimodal Sparse Coding
}

\author{
M.Nandakishore \\ MCA, M Tech \\ Assistant Professor, \\ Department of MCA, \\ Sri Venkateswara college of \\ Engineering \& Technology, \\ Chittoor.
}

\author{
T.M.Theja Sree \\ PG Scholar, \\ Department of MCA, \\ Sri Venkateswara college of \\ Engineering \& Technology, \\ Chittoor.
}

\author{
U.Lakshmi Priya \\ PG Scholar, \\ Department of MCA, \\ Sri Venkateswara college of \\ Engineering \& Technology, \\ Chittoor.
}

\begin{abstract}
Picture reranking is helpful for adjusting the presentation of content base picture seeks. In any case, reachable reranking estimations are compelled for two essential drivers: 1) the printed meta-data related with pictures is every now and again opposite with their real picture substance and 2) the uprooted visual highlights don't decisively demonstrate the semantic resemblances among pictures. Starting late, customer snap information has been used as a piece of picture reranking, for the reason that snaps have been introduced to incorporate unequivocally depict the essentialness of recouped pictures to interest request. Regardless, a critical circumstance for snap based systems is the need of snap data, in light of the fact that simply a bit number of web pictures has truly been tapped on by customers. Thusly, we hope to deal with this issue by gaging picture clicks. We propose a multimodal hyper chart learning-based small coding methodology for picture snap figure, and impact the procured snap information to the re positioning of pictures. We got a hyper diagram to set up a gathering of manifolds, where it have diverse highlights through a social event of weights. Separating, a graph that has an edge between two vertices, a hyper edge in a hyper chart join a course of action of vertices, and associates guarantee the close-by smoothness of the constructed lacking coding. An erratic change technique is then performed, and the weights of various modalities and the insufficient code are meanwhile gotten. Finally, voting philosophy is used to delineate the foreseen snap as a parallel event (snap or no snap), from the photos alike lacking codes. Thorough trial studies on a far reaching scale database including right around $330 \mathrm{~K}$ photos demonstrate the estimation of our technique for snap gage when differentiated and a couple of diverse schedules. Additional picture re positioning examinations on certifiable data exhibit the usage of snap guess is helpful to acculturating the presentation of surprising diagram based snap re positioning counts.
\end{abstract}

\section{General Terms}

Annotation process, Concatenating technique

\section{Keywords}

Picture reranking, snap, manifolds, pitiful codes.

\section{INTRODUCTION}

Due to the massive number of pictures on the web, picture look for development has transformed into a dynamic and testing investigation subject. All that much saw picture web pursuit devices, for instance, Bing, Yahoo and, by and large Google use content based meta-data included in the including substance, titles, subtitles, and URLs, to document web pictures. Regardless of the way that the execution of substance based picture recuperation for a few interests is sufficient, the precision and profitability of the recouped results could at present be upgraded on a very basic level.

One important issue influencing execution is the clutters between the genuine substance of picture and the printed data on the site page. One procedure used to deal with this issue is picture re-situating, in which both abstract and visual information are joined to return improved results to the customer. The situating of pictures in light of a substance based chase is seen as a sensible gage, however with fuss. Removed visual information is then used to re-rank related pictures to the most noteworthy need on the summary.

Most existing re-situating schedules use an instrument known as pseudo-hugeness feedback (PRF), where a degree of the top-situated pictures are thought to be apropos, and therefore used to create a model for re-situating. This is instead of significance data, where customers unequivocally give feedback by naming the top results as positive or negative. In the request based PRF framework, the top-situated pictures are seen as pseudo-positive, and low-situated pictures saw as pseudo-negative specimens to set up a classifier, and after that re-rank, moreover get this pseudo-positive and pseudonegative picture system to add to a gathering based resituating count.

The issue with these methods is the relentless nature of the got pseudo-positive and pseudo-negative pictures is not guaranteed. PRF has in like manner been used as a piece of outline based re-situating and Bayesian visual re-situating. In these schedules, low-rank pictures are progressed by getting backing from related high-rank pictures. Then again, these methods are limited by the way that unessential high-rank pictures are not downsized. Along these lines, both unequivocal and irrefutable re-situating procedures encounter the evil impacts of the precariousness of the first situating summary, since the artistic information can't definitely depict the semantics of the inquiries.

Instead of related abstract information, customer snaps have starting late been used as a more strong measure of the relationship between the inquiry and recouped things, since snaps have been demonstrated to more decisively reflect the noteworthiness drove an eye-taking after examination to watch the relationship between the clicked associations and the significance of the target pages, while inspected the effect of reordering web filed records in light of explore chase practicality. 
Because of picture looking, snaps have wound up being greatly tried and true; $84 \%$ of clicked pictures were critical differentiated with $39 \%$ relevance of files found using a general web look. Considering this, Jain proposed a system which uses clicks for request ward picture looking for. Regardless, this framework just investigates snaps and slights the visual highlights which may upgrade the recouped picture relevance to the request. In another study, Jain and Varna proposed a Gaussian backslide model which straight forwardly associate the snaps and distinctive visual highlights into a long vector. Tragically the contrasting characteristics of different visual highlights were not pondered. As demonstrated by business web crawler examination reports, only $15 \%$ of web pictures are clicked by web customers. This nonappearance of snaps is an issue that makes convincing snap based re-situating striving for both speculative studies and certifiable execution. With a particular deciding objective to deal with this issue, we get small coding to predict click information for web pictures.

Small coding is a surely understood sign get ready system and performs well in various applications, e.g. signal entertainment, signal decay, and sign removing. Yet orthogonal bases like Fourier or Wavelets have been for the most part grasped, the latest example is to get an over complete reason, in which the amount of reason vectors is more important than the dimensionality of the information vector. A sign can be depicted by a course of action of over complete bases using a bit number of nonzero segments. This reasons high inadequately in the change zone; however various applications oblige this negligible representation of signs. In PC vision, signs are picture highlights, and pitiful coding is held onto as a compelling system for highlight changing. It has been comprehensively used as a piece of different applications, for instance, picture plan, face affirmation, picture annotation, and picture recovery.

\section{SYSTEM ARCHITECTURE}

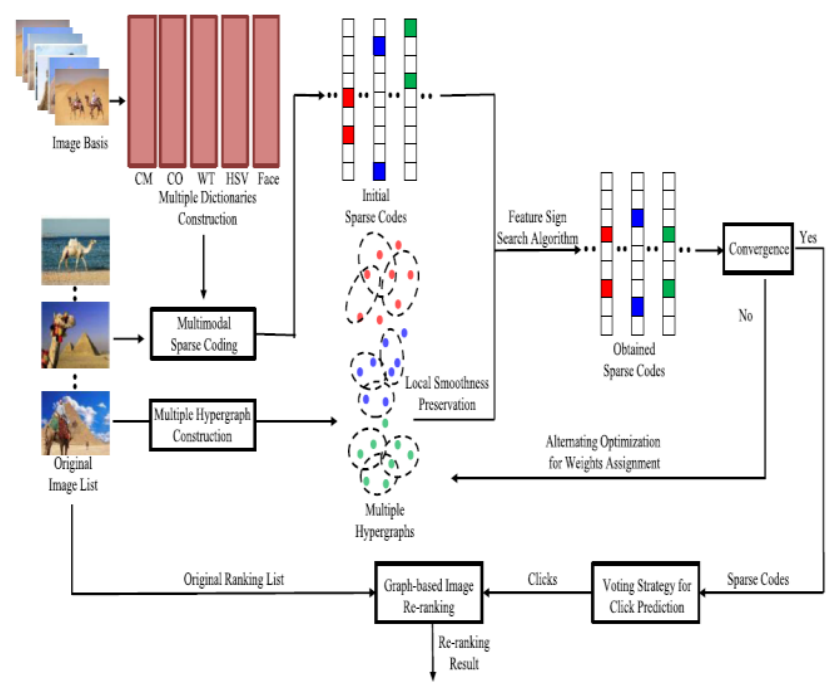

Figure 2: System Architecture diagram

In this graph, utilizing inadequate coding we develop multimodal diagrams and utilizing this term, we can clarify the snaps for the new information pictures and after that repositioning.

\subsection{Annotation Process}

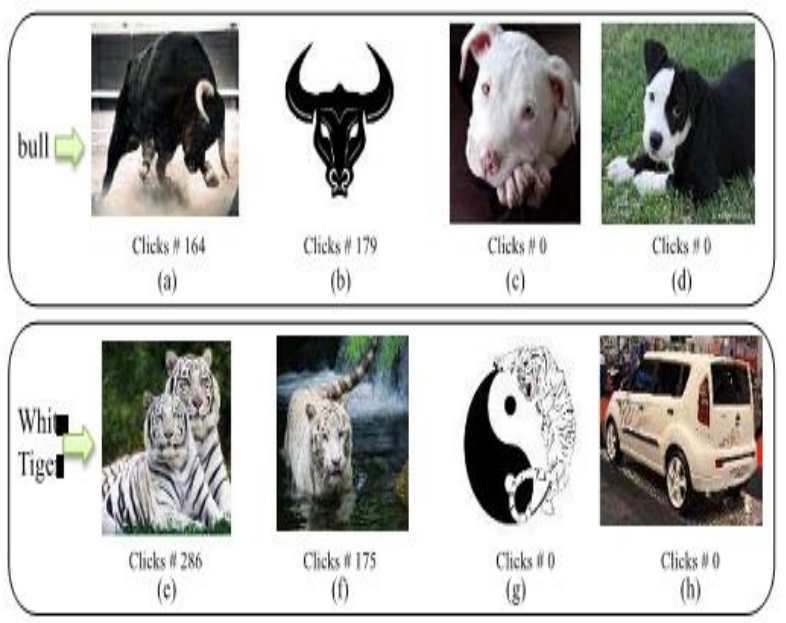

Fig: Similarities in images

Initially, we develop a web picture base with related snap annotation, gathered from a business internet searcher. As demonstrated in Fig. 1, the internet searcher has recorded snaps for every picture. Fig. 1(a), (b), (e), and (f) demonstrate that the pictures with high snaps are unequivocally applicable to the inquiries, while Fig. 1(c), (d), (g), and (h) exhibit nonsignificant pictures with zero ticks. These two parts shape the picture bases.

We successfully use web search tool inferred pictures commented with snaps, and effectively anticipate the snaps for new information pictures without snaps. In light of the got clicks, we re-rank the pictures, a methodology which could be advantageous for enhancing business picture looking.

\subsection{Concatenating Technique}

Second, we propose a novel system named multimodal hyper chart learning-based scanty coding. This strategy utilizes both early and late combination in multimodal learning. By at the same time taking in the meager codes and the weights of distinctive hyper charts, the execution of inadequate coding performs essentially. We consider both early and late combination in the proposed target capacity. The early combination is acknowledged by straightforwardly linking numerous visual highlights, and is connected in the scanty coding term. Late combination is proficient in the complex learning term. For web pictures without snaps, we actualize hyper chart figuring out how to develop a gathering of manifolds, which saves neighborhood smoothness utilizing hyper-edges. Dissimilar to a chart that has an edge between two vertices, an arrangement of vertices are joined by the hyper edge in a hyper-diagram. Normal chart based learning techniques generally just consider the pair shrewd relationship between two vertices, overlooking the higher-request relationship among three or more vertices. Utilizing this term can help the proposed system safeguard the nearby smoothness of the built inadequate codes.

\subsection{Optimization Process}

At long last, a rotating streamlining method is led to investigate the corresponding way of distinctive modalities. The weights of diverse modalities and the inadequate codes are all the while got utilizing this enhancement methodology. A voting system is then embraced to anticipate if an info picture will be clicked or not, in light of its meager code. The 
acquired snap is then coordinated inside a diagram based learning structure to attain to picture re-positioning.

\section{EXISTING SYSTEM}

Most existing re-situating systems use a gadget known as pseudo-congruity feedback (PRF), where a degree of the topsituated pictures are thought to be essential, and in this manner used to gather a model for re-positioning. This is rather than congruity info, where customers unequivocally give feedback by denoting the top results as positive or negative. In the course of action based PRF method, the topsituated pictures are seen as pseudo-positive and low-situated pictures saw as pseudo-negative examples to set up a classifier, and thereafter re-rank. Hsu et al. moreover grasp this pseudo-positive and pseudo-negative picture method to develop a clustering based re-situating figuring.

\subsection{Disadvantages}

One essential issue influencing execution is the perplexes between the genuine substance of picture and the content construct data in light of the website page. The issue with these schedules is the reliability of the procured pseudopositive and pseudo-negative pictures is not guaranteed.

\section{PROPOSED SYSTEM}

In this task, we propose a novel procedure named multimodal hyper diagram learning-based sparse coding for snap desire, and apply the expected snaps to re-rank web pictures. Both procedures of in front of calendar and late mix of various highlights are used as a piece of the framework through three essential step

We add to a web picture base with related snap annotation, accumulated from a business web crawler. The web quest apparatus has recorded snaps for each photo. Demonstrate that the photos with high snaps are insistently correlated to the inquiries, while show non-imperative pictures with zero ticks. These two sections outline the photo bases.

We consider both early and late blend in the proposed target limit. The early mix is recognized by direct connecting different visual highlights, and is associated in the insufficient coding term. Late blend is capable in the complex learning term. For web pictures without snaps, we execute hyper chart making sense of how to add to a get-together of manifolds, which defends neighborhood smoothness using hyper edges. Not under any condition like a chart that has an edge between two vertices, is a plan of vertices related by the hyper edge in a hyper outline. Typical outline based learning strategies regularly simply consider the pair sagacious relationship between two vertices, sitting above the higher-demand relationship among three or more vertices. Using this term can help the proposed framework spare the area smoothness of the assembled meager codes.

Finally, a trading streamlining system is coordinated to examine the complementary method for different modalities. The weights of unmistakable modalities and the insufficient codes are in the meantime got using this upgrade system. A voting technique is then gotten to predict if an information picture will be clicked or not, in perspective of its pitiful code.

\subsection{Advantages}

We enough utilize web crawler induced pictures cleared up with snaps, and viably predict the snaps for new incorporate pictures without snaps. Considering the got clicks, we re-rank the photos, a technique which could be valuable for upgrading business picture looking.

Second, we propose a novel technique named multimodal hyper chart learning-based inadequate coding. This strategy uses both early and late mix in multimodal learning. By at the same time taking in the lacking codes and the weights of diverse hyper diagrams, the execution of inadequate coding performs fundamentally. We consider both early and late combination in the proposed target capacity. The early combination is acknowledged by specifically connecting numerous visual highlights, and is connected in the inadequate coding term. Late combination is refined in the complex learning term. For web pictures without snaps, we execute hyper chart figuring out how to develop a gathering of manifolds, which protects nearby smoothness utilizing hyperedges. Not at all like a chart that has an edge between two vertices, an arrangement of vertices are joined by the hyper edge in a hyper-diagram. Normal chart based learning techniques typically just consider the pair astute relationship between two vertices, disregarding the higher-request relationship among three or more vertices. Utilizing this term can help the proposed technique protect the neighborhood smoothness of the developed meager codes.

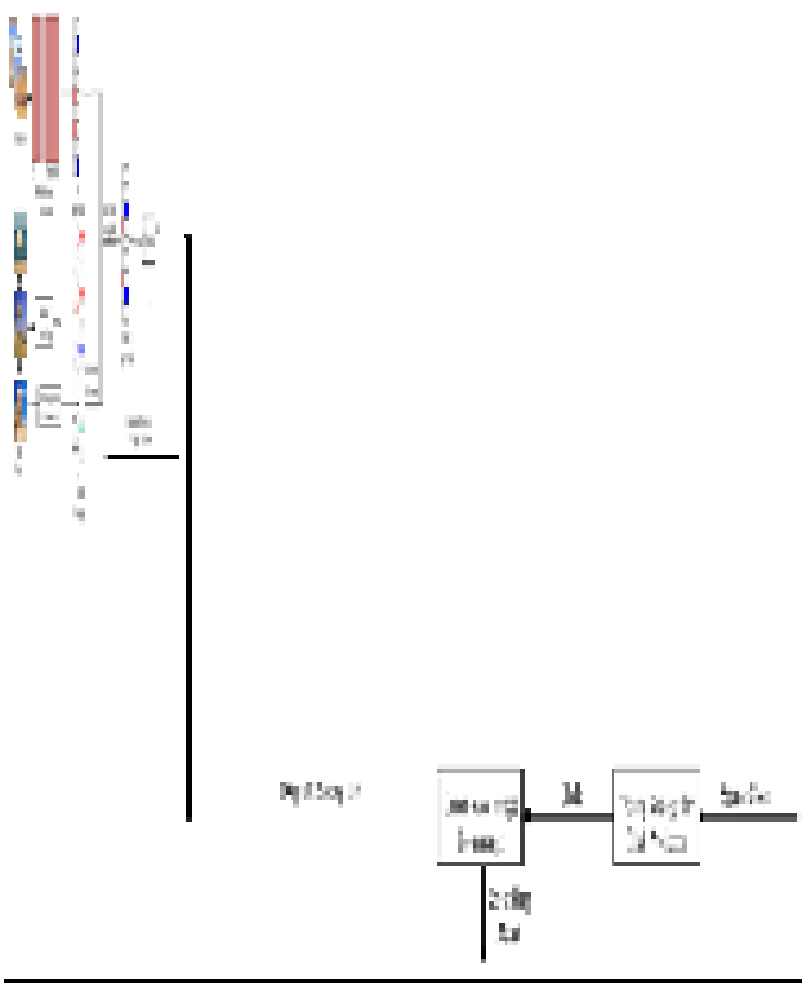

Chart based learning routines have been broadly utilized as a part of the fields of picture characterization, positioning and grouping. In these techniques, a diagram is manufactured by given information, where vertices speak to information tests and edges portray their likenesses. The Laplacian framework is developed from the diagram and utilized as a part of a regularization plan. The nearby geometry of the chart is saved amid the enhancement, and the capacity is powerfully smoothed on the diagram. However, a basic diagram based system can't catch higher- request data. Dissimilar to a basic chart, a hyper edge in a hyper diagram connects a few (two or more) vertices, and in this way catches this higher-request data. 


\section{RESULTS}

At long last, a rotating streamlining methodology is directed to investigate the reciprocal nature from every question to shape the picture base. The tests were directed under five contrast ent conditions, where the extent of information pictures changed in the scope of $5-25 \%$. As per the exploratory results, we watch that almost all the utilized routines viably enhance pattern relative results. Our technique, MHL, accomplished the best results for click forecast, with the hyper chart based system performing better than other single diagram based strategies. The higharrange data safeguarded by hyper chart development is useful to safeguarding nearby smoothness. Contrasted and a typical diagram, the utilization of the hyper chart can viably enhance technique was used to foresee whether the pictures would be clicked or not. We got the characterization exactness (\%) as an appraisal of the consequence of snap expectation. We utilized $75 \%$ pictures from every inquiry to frame the picture base. The tests were directed under five contrast ent conditions, where the extent of data pictures shifted in the scope of $5-25 \%$. As indicated by the trial results, we watch that about all the utilized techniques successfully enhance gauge near results. Our system, MHL, attained to the best results for click forecast, with the hyper diagram based technique performing better than other single chart based systems. The high-arrange data saved by hyper chart development is useful to protecting nearby smoothness. Contrasted and an ordinary diagram, the utilization of the hyper chart can viably enhance click expectation execution. Also, we watch that multimodality systems (MHL and MGL) beat single methodology strategies (SHL and SGL).

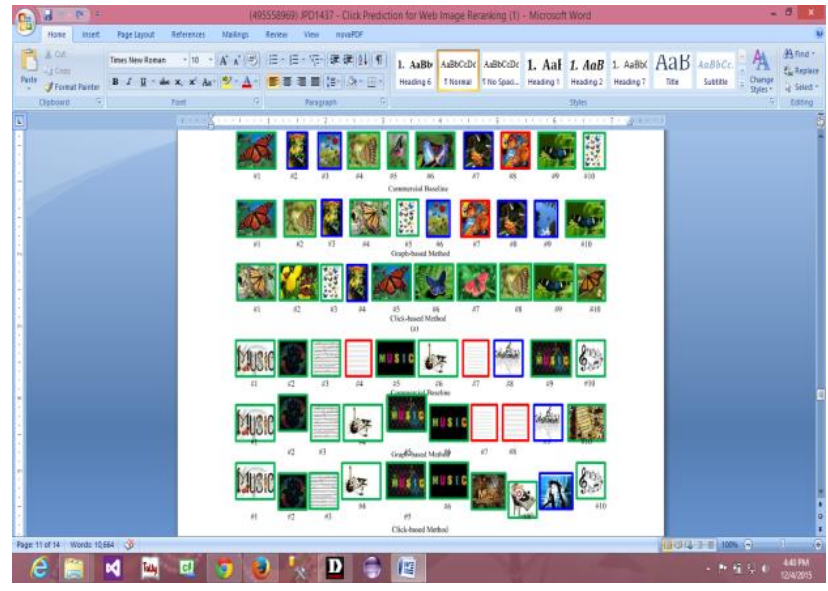

We watched that the characterization exactness of MHL increments from $64.8 \%$ to66.9\% when the span of the picture base increments from $10 \%$ to $90 \%$ demonstrating that a little picture base can antagonistically influence click expectation execution.

\section{CONCLUSION}

In this venture, we propose new multimodal hyper chart learning based meager coding system for the snap forecast of pictures. The got inadequate codes can be utilized for picture re-positioning by incorporating them with a diagram based construction. We embrace a hyper chart to manufacture a gathering of manifolds, which through a gathering of weights. Dissimilar to a diagram that has an edge between two vertices, an arrangement of vertices are associated by a hyper edge in a hyper chart. This helps safeguard the neighborhood smoothness of the built scanty codes. At that point, a substituting streamlining strategy is performed and the weights of diverse modalities and meager codes are at the same time acquired utilizing this enhancement methodology. At long last, a voting method is utilized to foresee the snap from the relating meager code. Exploratory results on certifiable information set shave exhibited that the proposed technique is viable in deciding snap forecast. Extra trial results on picture re-positioning propose that this strategy can enhance the outcomes returned by business web crawlers.

\section{ACKNOWLEDGEMENT}

We take this chance to thank our task guide Mr.M.Nanda Kishore for his important direction and for giving all the fundamental offices, which were essential in fruition of this undertaking.

\section{REFERENCES}

[1] Y. Gao, M. Wang, Z. J. Zha, Q. Tian, Q. Dai, and N. Zhang, "Less is more: Efficient 3D object retrieval with query view selection," IEEE Trans. Multimedia, vol. 13, no. 5, pp. 1007-1018, Oct. 2011.

[2] S. Clinchant, J. M. Renders, and G. Csurka, "Trans-media pseudo relevance feedback methods in multimedia retrieval," in Proc. CLEF, 2007, pp. 1-12.

[3] L. Duan, W. Li, I. W. Tsang, and D. Xu, "Improving web image search by bag-based reranking," IEEE Trans. Image Process., vol. 20, no. 11, pp. 3280-3290, Nov. 2011.

[4] B. Geng, L. Yang, C. Xu, and X. Hua, "Content-aware Ranking for visual search," in Proc. IEEE Conf. Comput. Vis. Pattern Recognit., Jun. 2010, pp. 3400-3407.

[5] B. Carterette and R. Jones, "Evaluating search engines by modeling the relationship between relevance and clicks," in Proc. Adv. Neural Inf. Process. Syst., 2007, pp. 1-9.

[6] G. Dupret and C. Liao, "A model to estimate intrinsic document relevance from the clickthrough logs of a web search engine," in Proc. ACM Int. Conf. Web Search Data Mining, 2010, pp. 181-190.

[7] G. Smith and H. Ashman, "Evaluating implicit judgments from image search interactions," in Proc. WebSci. Soc., 2009, pp. 1-3. 